\title{
Jingui Shenqi Pills Prevents and Treats Postmenopausal Osteoporosis via Regulating the BMP/Smad Signaling Pathway
}

\section{Qian Zhang}

Shandong University of Traditional Chinese Medicine

\section{Xu Yang}

Shandong University of Traditional Chinese Medicine

\section{$\mathrm{Ke} \mathrm{Ma}$}

Shandong University of Traditional Chinese Medicine

Yanan Zhang

Shandong University of Traditional Chinese Medicine

\section{Xu Tian}

Shandong University of Traditional Chinese Medicine

\section{Yuan Wang}

Shandong University of Traditional Chinese Medicine

Xiaochun Han

Shandong University of Traditional Chinese Medicine

Jin Wang

Shandong University of Traditional Chinese Medicine

\section{Shijun Wang}

Shandong University of Traditional Chinese Medicine

Huaxin Wang ( $\nabla$ whx215@163.com)

Shandong University of Traditional Chinese Medicine https://orcid.org/0000-0002-8293-4231

\section{Research}

Keywords: Traditional Chinese Medicine, Jingui Shenqi pills, Postmenopausal Osteoporosis, BMP/Smad signalling Path

Posted Date: July 7th, 2021

DOI: https://doi.org/10.21203/rs.3.rs-669680/v1

License: (c) (1) This work is licensed under a Creative Commons Attribution 4.0 International License. 



\section{Jingui Shenqi pills Prevents and Treats Postmenopausal osteoporosis via Regulating the BMP/Smad Signaling Pathway}

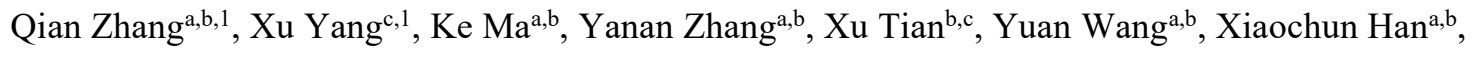
Jin Wang c, Shijun Wang,a,b, Huaxin Wanga,b,*

a. College of Traditional Chinese Medicine, Shandong University of Traditional Chinese Medicine, Jinan, Shangdong Province 250355, China

b. Shandong Co-Innovation Center of Classic TCM Formula, Shandong University of Traditional Chinese Medicine, Jinan, Shangdong Province 250355, China

c. Shandong University of Traditional Chinese Medicine, Jinan, Shangdong Province 250355, China

Running title: Jingui Shenqi pills in the Treatment of postmenopausal osteoporosis

\section{Address Correspondence to:}

Dr Huaxin Wang, Shandong Co-Innovation Center of Classic TCM Formula, College of Traditional Chinese Medicine, Shandong University of Traditional Chinese Medicine, No 4655, University Road, Changqing District, Jinan, Shandong 250355, PR China, Tel/Fax: +86-53189628077,Email: whx215@163.com;

Or Dr Shijun Wang, Shandong Co-Innovation Center of Classic TCM Formula, College of Traditional Chinese Medicine, Shandong University of Traditional Chinese Medicine, No 4655, University Road, Changqing District, Jinan, Shandong 250355, PR China, Tel/Fax+86-53189628077, Email: pathology@163.com.

${ }^{1}$ Qian Zhang and $\mathrm{Xu}$ Yang contributed equally to this work. 


\begin{abstract}
Background: Jingui Shenqi ( JGSQ for short) pills is a traditional Chinese medicine formula, which has the functions of warming and tonifying kidney-yang, generating essence and filling marrow, warming tendons and veins and bones, and improving bone mineral density. The aim of this study was to investigate the effect and mechanism of JGSQ pills in preventing and treating postmenopausal osteoporosis.
\end{abstract}

Methods: Twelve-week-old SPF female SD rats $(n=48)$ were used in this study. Following the ovariectomy operation $(n=40)$, the rats were randomly divided into the model group, (high, medium, and low dose groups) treated with Jingui Shenqi pills and estradiol group. Recorded the weight gain of rats and calculated the uterine coefficient; To detect the expression of serum calcium, phosphorus, ALP and OPG; HE staining was used to detect the pathological changes of femur; In all the groups, Micro-CT was used for detection of bone mineral density and bone microstructure; and gene expression of BMP-2, Smad1, and Runx2 in rat bone tissue was determined by RT-PCR and Western Blot methods.

Results: Compared with the sham operation group, rats in the model group had the highest increase in body weight and the lowest uterine coefficient. Each of the treatment groups had a modest increase in weight gain. Micro-CT and HE staining showed a decrease in bone mineral density in the model group with shorter, thinner, broken, and osteoporotic trabeculae of the femur. The bone mineral density in Jingui Shenqi pills treatment groups had a significant increase with an improved bone microstructure and intact bone trabecular as compared to the model group. Serum ALP in the model group was significantly higher than in the sham operation group but the serum calcium, phosphorus and OPG was significantly lower. The Jingui Shenqi pills treatment groups and the estradiol treated group had lower serum ALP and increased serum calcium, phosphorus and OPG.There was decreased gene expression of BMP-2, Smad1, and Runx2 in the bone tissue of the model group compared to the sham operation group. BMP-2, Smad1, and Runx2 gene expression in Jingui Shenqi pills treated group and estradiol treated group was significantly higher than that of the model group. 
Conclusion: Jingui Shenqi pills improve the microstructure of bone tissue and increase bone mineral density thus resolving osteoporosis. This is achieved by regulating BMP/Smad signaling pathway and increasing the expression of osteogenic factors BMP-2, Smad1, and Runx2.

Keywords Traditional Chinese Medicine; Jingui Shenqi pills; Postmenopausal Osteoporosis; BMP/Smad signalling Pathway.

\section{Graphical abstract:}

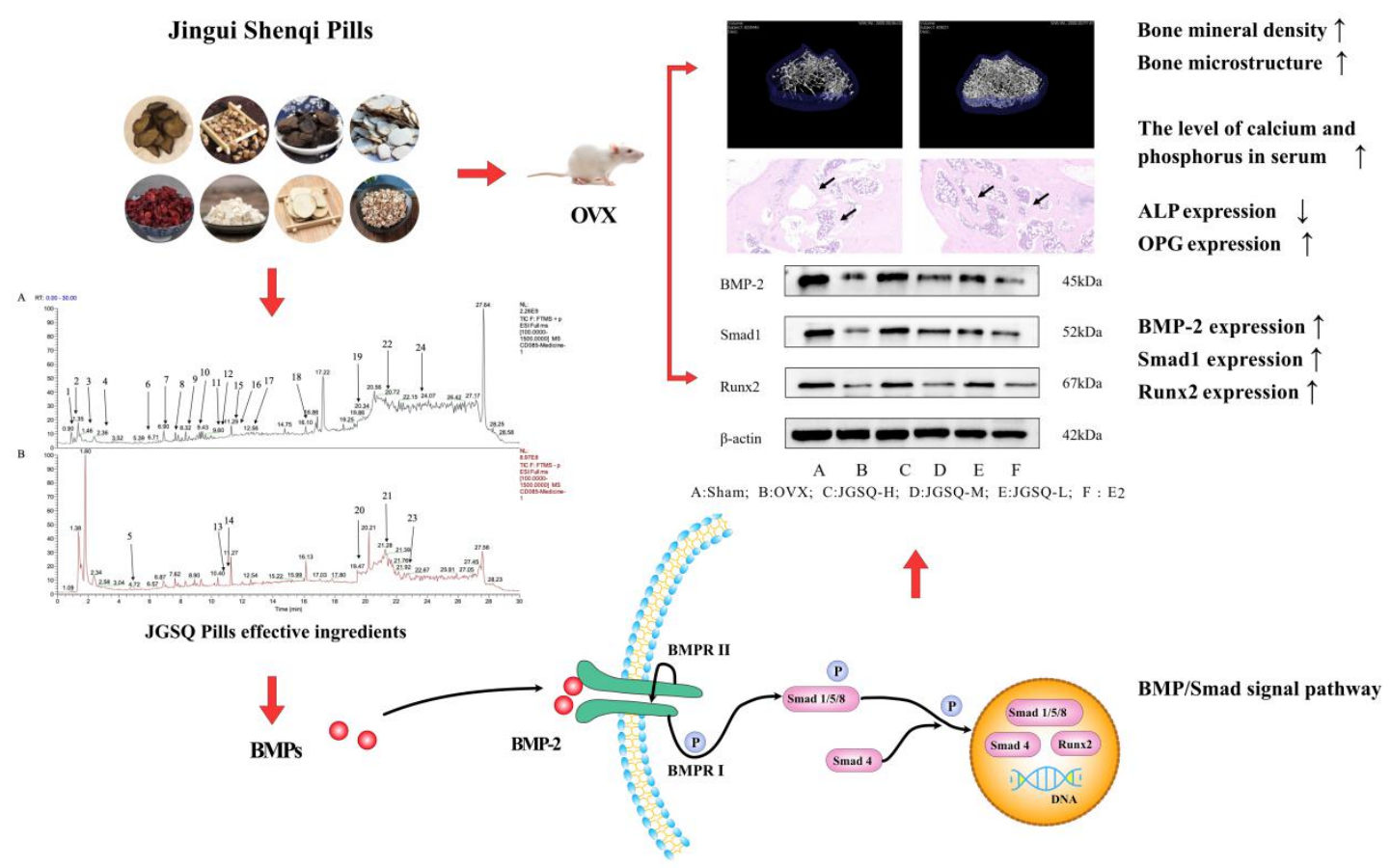




\section{Introduction:}

Osteoporosis is a metabolic disease that frequently occurs in elderly and postmenopausal women. It is mainly characterized by damage of the bone tissue microstructure, decrease of bone mineral density, and increased risk of fractures [1] [2]. Environmental and genetic factors accelerate the occurrence and development of osteoporosis, of which the imbalance of bone remodeling caused by reduced bone formation is a key factor in the occurrence of osteoporosis[3]. Statistics show that worldwide, 1,000 people are fractured due to OP every hour, and there are about 900 million fractures each year[4], of which the incidence of postmenopausal osteoporosis is about $30 \%$ in people over 50 years of age[5], and the resulting fractures are the main cause of middle-aged and elderly visits, which seriously affect the physical and mental health of middleaged and elderly people, bring a huge burden to families and society, and become an important public health problem currently faced .

Modern medicine believes that the dynamic balance of osteoblasts and osteoclasts is the key to maintain bone remodeling, thereby maintaining the balance of bone metabolism[6]. Bone remodeling is regulated by various factors, such as estrogen levels, intestinal bacteria, oxidative stress, and inflammatory responses[7]. At present, the commonly used anti-osteoporosis drugs in clinical practice include bisphosphonates, parathyroid hormone and hormone replacement therapy. However, while exerting the role of targeted therapy, the above drugs ignore the integrity of the body. Long-term or high-dose administration will increase the risk of cardiovascular disease, cervical cancer, kidney stones and gastrointestinal diseases[8]. Therefore, it is important to continue exploring for safe and effective treatment.

Traditional Chinese medicine seeks the root cause of the disease, pays attention to the occurrence, development, and disease changes to bring a balance to yin and yang. Traditional Chinese medicine has the advantage of minimal side effects and high efficacy in the treatment of postmenopausal osteoporosis. The multiple compounds in a traditional Chinese medicine preparation act on multi-targets and multi-pathways to promote bone formation. Traditional Chinese medicine also relieves the bone pain associated with osteoporosis[9]. Traditional Chinese medicine theories, postulate that the kidney controls bone marrow generation by regulating bone metabolism. Jingui Shenqi pills (JGSQ for short) are a classic prescription for warming and act 
as a tonic for kidney yang which was first published in the synopsis of the Golden Chamber. It is composed of dried Rehmannia glutinosa, yam, Cornus officinalis, Poria, alisma, moutan, aconite, and cassia twig. Currently, it is clinically used in the treatment of kidney yang deficiency[10] [11] [12].

Research has shown Jingui Shenqi pills relieve osteoporosis-associated bone pain[13]. Additionally, long-term treatment with Jingui Shenqi pills in postmenopausal osteoporosis resulted in a similar increase in bone mineral density as alendronate[14]. Jingui Shenqi pills were also shown to increase estrogen in in vivo studies carried out in ovariectomized rats. Jingui Shenqi pills also improved osteoblast vitality, ALP activity, and Collagen I protein expression[15]. Jingui Shenqi pills were also shown to promote bone differentiation in an in vitro study, by promoting the expression of ALP, RUNX2, and OCN[16]. However, the mechanism of Jingui Shenqi pills in preventing and treating PMOP has not been fully elucidated. Current studies have shown that the mechanism of traditional Chinese medicine in preventing and treating postmenopausal osteoporosis is related to the BMP/Smad signaling pathway, which can regulate all processes of osteoblast proliferation, differentiation, and mineralization, and its abnormal function can cause decreased bone mass and reduced bone formation, leading to osteoporosis[17] [18]. The BMP/Smad signaling pathway can regulate downstream osteoblast transcription factors, promote bone formation, and increase bone mineral density[19] [20] [21].

Therefore, in this experiment, ovariectomy was used to duplicate the postmenopausal osteoporosis model to observe the effect of Jinkui Shenqi Pills on calcium, phosphorus and bone formation markers in the serum of OVX rats; HE staining was used to observe the pathological changes of the femur; micro-CT was used to detect the bone mineral density and bone microarchitecture of rats; qRT-PCR and Western blot were used to detect the gene expression of BMP-2, Smad1 and Runx2 in the femur of rats to further elucidate the mechanism by which Jinkui Shenqi Pills improved osteoporosis in OVX rats. It further enriches the theory of "kidney main bone" and provides theoretical basis and scientific basis for the prevention and treatment of osteoporosis by traditional Chinese medicine.

\section{Materials and methods}




\subsection{JGSQ pills preparation}

All the traditional Chinese medicines of JGSQ were purchased from Shandong Xinzhonglu Hospital (Jinan, China). The preparation of JGSQ is composed of eight herbal medicines as follows: Rehmannia glutinosa 24g, Dioscorea zingiberensis 12g, Cornus officinalis 12g, Poria 9g, alisma alisma $9 \mathrm{~g}$, moutan bark $9 \mathrm{~g}$, aconite $3 \mathrm{~g}$, and cassia twig $3 \mathrm{~g}$. The herbal materials were decocted in eight volumes of distilled water for 1 hour. A filtrate was then obtained by double filtration. The filtrate was then concentrated to a high dose of $1.7 \mathrm{~g} / \mathrm{ml}$ (twice the clinical dose), a medium dose of $0.85 \mathrm{~g} / \mathrm{ml}$ (clinically used dose), and a low dose of $0.425 \mathrm{~g} / \mathrm{ml}$ (half the clinical dose). Medium dose is the original amount of classical prescription.

\subsection{Qualitative analysis for ingredients in JGSQ standard decoction}

To a plant decoction filtrate of $200 \mu 1,1 \mathrm{~mL}$ of methanol: water $(8: 2 \mathrm{~V}: \mathrm{V})$ was added. The solution was mixed by vortexing and centrifugation was done at $4{ }^{\circ} \mathrm{C}(20000 \mathrm{xg}, 10$ minutes), The supernatant was filtered with a $0.22 \mu \mathrm{m}$ membrane, and the filtrate was analyzed by HPLC. Ion source: electrospray ionization source (ESI); Scanning mode: positive and negative ion switching scanning; Detection method: Full mass/dd-MS2; Resolution: 70000(full mass), 17500(dd-MS2); scanning range: $100.0-1500.0 \mathrm{~m} / \mathrm{z}$, Spray Voltage:3.8kV(Positive), Capillary Temperature: $300^{\circ} \mathrm{C}$. An RP-C18 column $(150 \times 2.1 \mathrm{~mm} 1.8 \mathrm{um}$, Welch $)$ was used for all analyses and maintained at $30{ }^{\circ} \mathrm{C}$ at a flow rate of $0.3 \mathrm{ml} / \mathrm{min}$. , The mobile phase comprised $0.1 \%$ formic acid (v/v) (A) and acetonitrile (B) (Table 1).

\subsection{Animals model establishment and drug administration}

12 week-old female Sprague Dawley rats $(\mathrm{n}=48)$ were obtained from the Laboratory Animal Center of Shandong University of Traditional Chinese Medicine (Shandong China). They were maintained in a $12 \mathrm{~h}$ light-dark cycle, at a controlled temperature $\left(22-24^{\circ} \mathrm{C}\right)$ and humidity $(50-$ $60 \%$ ). Standard laboratory rodent diet and water were provided ad libitum. The rats were subjected to adaptive feeding for three days before the experiment. Complete bilateral ovariectomy was then carried out in all rats $(n=40)$ except the rats allocated to the sham group ( a sham operation involving removal of the same amount of fat near the ovary was carried out). Five days after the operation, the rats were allocated to six groups of 8 rats as follows: sham (shamoperated rats + saline), OVX (OVX rats + saline), high-dose JGSQ pills (OVX + JGSQ-H, 1.7 $\mathrm{g} / \mathrm{kg} / \mathrm{d})$, medium-dose JGSQ pills(OVX + JGSQ-M, $0.85 \mathrm{~g} / \mathrm{kg} / \mathrm{d})$, Low-dose JKSQ pills(OVX + JGSQ-L, $0.425 \mathrm{~g} / \mathrm{kg} / \mathrm{d}$ ), estradiol (OVX $+\mathrm{E}_{2}, 0.1 \mathrm{~g} / \mathrm{kg}$ body weight).The medium dose of JGSQ and Estradiol was equivalent to clinically used doses.This experiment was reviewed by the Ethical 
Review Committee of Experimental Animal Welfare of Shandong University of traditional Chinese Medicine, and met the welfare and ethical requirements of experimental animals and animals (Batch number: SDUTCM2018112901).

\subsection{Serological index detection}

Analysis of calcium and phosphorus (C004-2, C006-1-1, Nanjing Jiancheng) in serum was done using an automatic biochemical analyzer (AU2700, Beckman Technology Co., Ltd.).Serum markers of bone formation: ALP (A059-2, Nanjing Jiancheng) and OPG(E-EL-R0050c, Wuhan Elabscience) levels in the serum were determined using ELISA kits per the manufacturer's instructions. The bone turnover markers were assessed at $450 \mathrm{~nm}$ using an enzyme-linked immune detector (American Boteng instrument Co., Ltd. VT 05404-0998).

\subsection{Hematoxylin and eosin (H\&E) staining}

The left femur bones were collected, fixed in $10 \%$ neutral formaldehyde for $72 \mathrm{~h}$, then removed and decalcified in $10 \%$ ethylenediaminetetraacetic acid solution $(\mathrm{pH} 7.4)$ at $4^{\circ} \mathrm{C}$ for 4 weeks. Paraffin was then used to embed the fixed samples. The samples were then cut into $4 \mu \mathrm{m}$ slices which were stained with H\&E stain and observed under a light microscope.

\subsection{BMD and Micro-CT examination}

Local bone mineral density $\left(\mathrm{g} / \mathrm{cm}^{2}\right)$ and trabecular microstructure $(\mathrm{BV} / \mathrm{TV}, \mathrm{BS} / \mathrm{BV}, \mathrm{Tb} . \mathrm{Th}$, Tb.N, SMI, Tb.Sp and BMD ) of the right distal femur were measured and analyzed by BrukerMicro CT (NEMO, Pingsheng Medical Technology Co., Ltd.).Following the CT scan, the cortical and trabecular bones of the femur were analyzed by Skyscan NRecon software. The 3D and 2D sections of femoral cortical and trabecular bone were reconstructed.

\section{7 qRT-PCR}

Total RNA was extracted from the bone tissue with Vazyme R401-01RNA isolator per the manufacturer's instructions. Reverse transcription was then carried out using Vazyme R323-01kit. Primer sequences were designed based on GenBank sequences with Primer Premier 5.0 software 
(Table 2) by Shanghai Shenggong Co., Ltd., China. ACTB was used as an internal control. SYBRGreen PCR kit (Vazyme Q711-02/03) was used to prepare the reaction master mix, Fluorescencequantitative PCR (Bio-Rad, USA) was used for mRNA amplification and quantification. The reaction conditions were: denaturation at $95{ }^{\circ} \mathrm{C}$ for $30 \mathrm{~s}$, followed by 40 cycles of denaturation at $95{ }^{\circ} \mathrm{C}$ for $5-10 \mathrm{~s}$, annealing at $60^{\circ} \mathrm{C}$ for $30 \mathrm{~s}$, and separation at $95^{\circ} \mathrm{C}$ for $15 \mathrm{~s}$, $60^{\circ} \mathrm{C}$ for $60 \mathrm{~s}$ and $95^{\circ} \mathrm{C}$ for $15 \mathrm{~s}$. Relative gene expression was determined using the $2^{-\Delta \Delta \mathrm{ct}}$ method.

\subsection{Western blot}

The total protein was extracted from bone tissue using RIPA lysis buffer (P0013B, Beyotime) after pulverizing in liquid nitrogen. The concentration of the protein was determined using a BCA protein kit (G2026, Servicebio). The protein was then mixed with loading buffer and boiled at $100{ }^{\circ} \mathrm{C}$ for $5 \mathrm{~min}$ to denature it. $30 \mu \mathrm{g}$ of total protein was separated by $10 \%$ SDS-PAGE and transferred into $0.45 \mu \mathrm{m}$ polyvinylidene fluoride (A29280264, GE Healthcarelifescience) membranes. After blocking with 5\% nonfat dried milk for one and a half hours, membranes were incubated with primary antibodies at room temperature: rabbit anti-BMP-2 (Ab14933, Abcom), rabbit anti-Smad1 (10429-1-AP, Proteintech), and rabbit anti-Runx2 (Bs-1134R, Bioss) respectively for 2 hours. The membranes were then incubated with goat anti-rabbit IgG (LY192274, Servicebio) as the secondary antibody for $1 \mathrm{~h}$ at room temperature. The blots were developed with ECL reagent (B500023, Proteintech). The total protein was compared with $\beta$-actin as an internal reference, and the gray values of internal reference and target bands were quantified by Image-ProPlus6.0 software.

\subsection{Statistical analysis}

SPSS 22.0 (SPSS Inc., Chicago, IL, USA) was used to perform the statistical analyses and the data were expressed as mean \pm SEM. Differences between two groups were compared by $t$-test, while the comparison between three or more groups were performed through one-way analysis of variance (ANOVA) followed by Dennett's test in order to detect intergroup differences. $P<0.05$ was considered as statistically significant.

\section{Results}




\subsection{Identified compounds in JGSQ pills}

The components of the JGSQ (Salsolinol, choline, uracil, gallic acid, caffeic acid, et al) filtrate was verified by Q-Orbitrap high-resolution liquid chromatography-mass spectrometry under optimized conditions. The main chemical components in JGSQ freeze-dried powder were detected by comparing with the retention time and UV spectrum of the reference standard. A total of 24 peak signals were obtained. The chemical components were also identified(Table 3 and Fig $1)$.

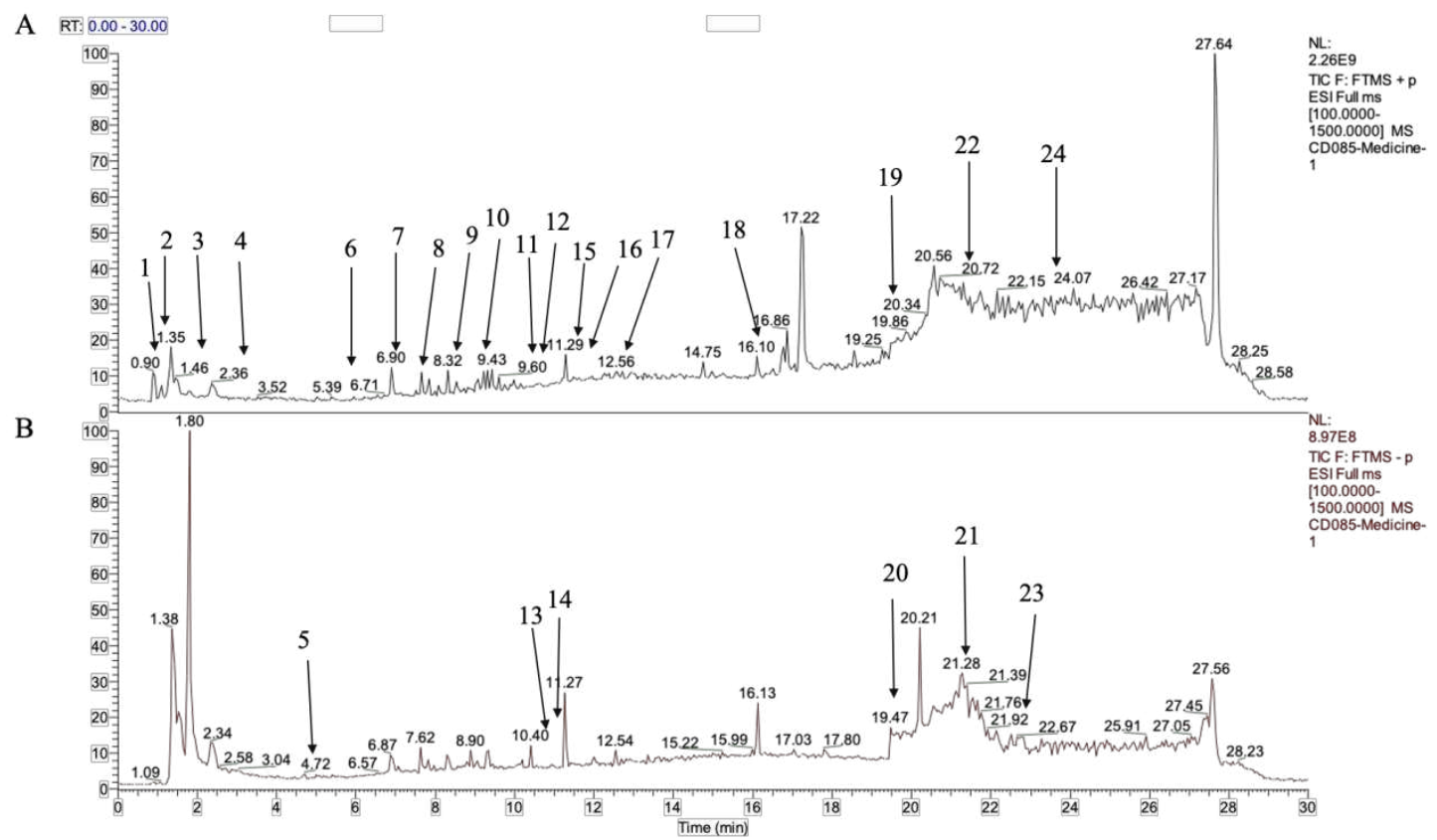

Figure.1 Total ion flow patterns of JGSQ pills in positive (A) and negative ion (B) modes.

\subsection{The general condition of rats}

Rats in the Sham group, had soft, shiny hair, were sensitive to activity. Rats in the OVX group had a significant decrease in activity as compared to the other treatment groups. The body weight of the rats was recorded weekly during the 3 months. Compared with the Sham group, the OVX group had the highest weight gain and lowest uterine coefficient, while JGSQ-H, JGSQ-M, JGSQ-L, and E2 groups recorded a lower weight gain (Fig.2A-2B), and an insignificant change in the uterine coefficient (Fig.2C). 

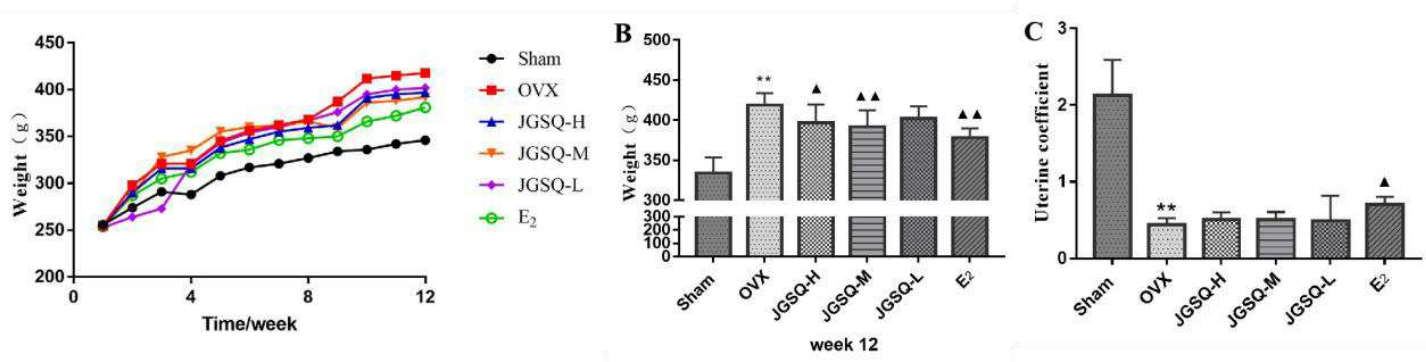

Figure.2 The general condition of rats. A. Effect of Jingui Shenqi pills on rats body weight. B. Body weight of rats in each group at Week 12. C. Effect of Jingui Shenqi pills on uterine coefficient different rat groups. *represents a comparison with the sham group, ${ }^{*} P<0.05,{ }^{* *} P<$ $0.01 ; \triangle^{\star}$ represents a comparison with OVX group, ${ }^{\mathbf{\Delta}} P<0.05, \stackrel{\Delta}{\Delta} P<0.01$.

\subsection{Biochemical index}

After 3 months of treatment, the serum levels of calcium and phosphorus in the OVX group were significantly lower than in the Sham group. However, the serum levels in the JGSQ-H group were significantly higher than in the OVX group. There was no significant difference between the treatment groups (Fig.3A-3B).

\subsection{Serum bone formation markers}

Compared with the Sham group, the serum ALP levels in the OVX group were significantly higher, and the serum OPG was significantly lower. The JGSQ-H, JGSQ-M, JGSQ-L, and E2 treated groups had a decrease in the levels of ALP and an increase in serum OPG (Fig.3C-3D). 

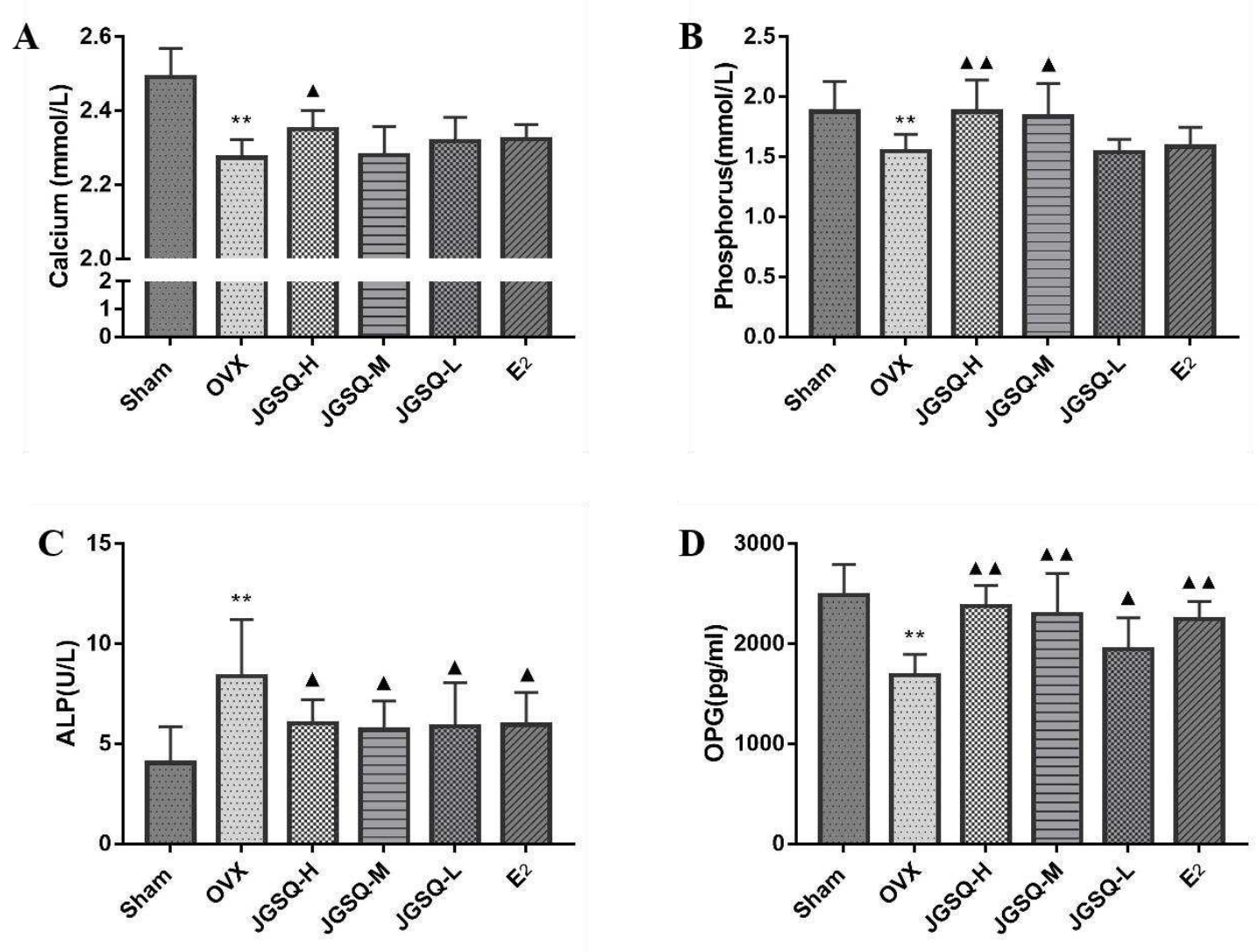

Figure.3 Effect of Jingui Shenqi Pills on Serum Parameters in Ovariectomized Rats. A,B.

Effect of Jingui Shenqi pills on calcium and phosphorus in serum of ovariectomized rats. C,D. Effect of Jingui Shenqi pills on serum ALP and OPG in ovariectomized rats. *represents a comparison with the sham group, ${ }^{*} P<0.05,{ }^{*} P<0.01 ;{ }^{\Delta}$ represents a comparison with OVX group, $\mathbf{\wedge} P<0.05, \boldsymbol{\wedge} \mathbf{\Delta} P<0.01$.

\subsection{Histopathological analysis}

The results of HE staining showed that the bone tissue structure in the Sham group was stable, the trabecular was dense and tidy, and the cortical and cancerous bones could be seen. In the OVX group, the bone tissue structure was seriously damaged, with a reduced trabecular volume and thickness, and a damaged medullary cavity space. Treatment with Jingui Shenqi pills showed an improvement in the bone microstructure, increased bone trabeculae, and an increase in the bone connection (Fig.4). 


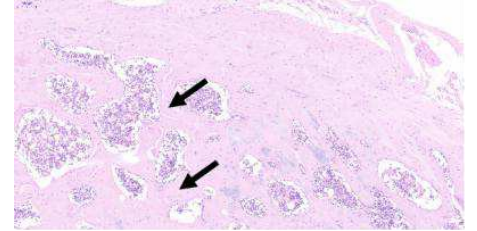

Sham

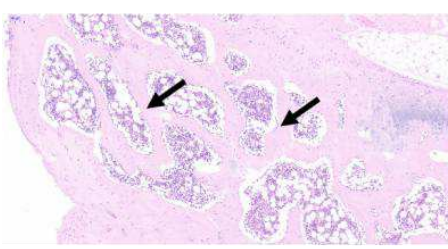

JGSQ-M

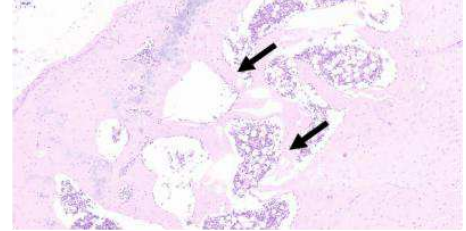

OVX

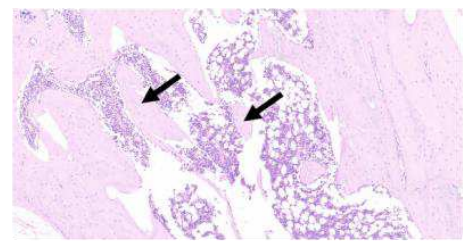

JGSQ-L

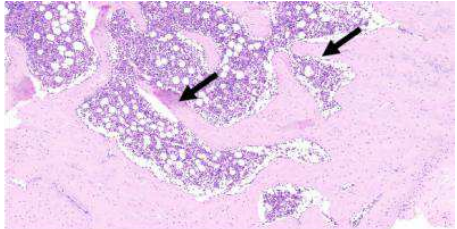

JGSQ-H

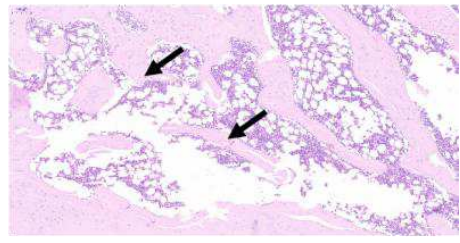

$\mathbf{E}_{2}$

Figure.4 Effect of Jingui Shenqi Pills on Pathological Changes of Femur in Ovariectomized

Rats.Representative photographs of H\&E stained femoral sections from each group $(\times 100)$.Arrow (black): bone.

\subsection{BMD and bone microstructure analysis}

The results of the previous preliminary experiment showed that the bone mineral density of the JGSQ-L group was not significantly improved compared with the model group, so only the JGSQ-H and JGSQ-M groups were tested. Compared with the Sham group, BMD decreased significantly in the OVX group but had a significant increase in the JGSQ-M group. Threedimensional imaging of the femur of rats in the Sham group showed the trabecular as being moderate in size, thickness, compact arrangement, and had a good bone connection, while the OVX group, had shorter, thinner, fewer, sparse, and fractured trabecular, with loose shape. After treatment with Jingui Shenqi pills, the bone microstructure improved significantly and had an intact trabecular structure (Fig.5). 

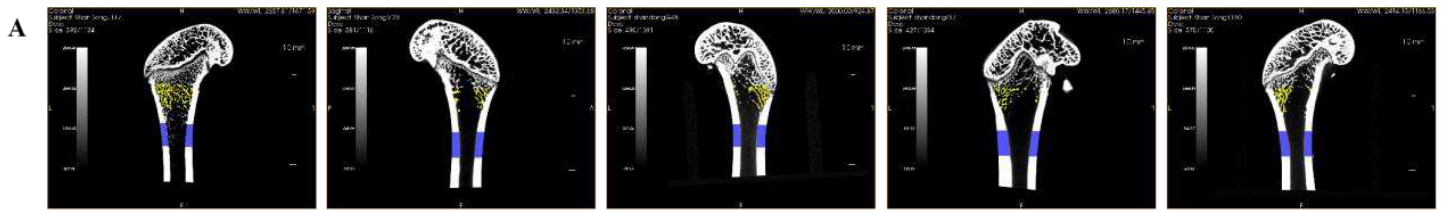

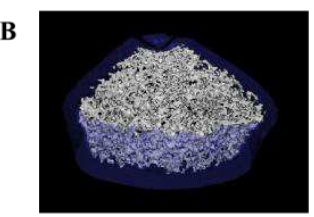

Sham

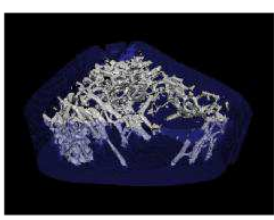

OVX

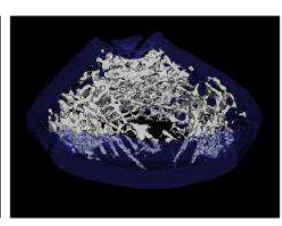

JGSQ-H

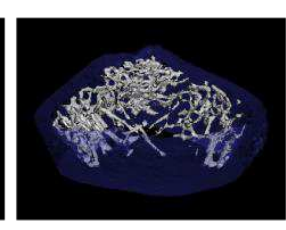

JGSQ-M

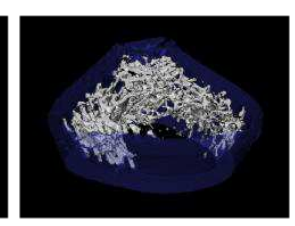

$\mathbf{E}_{2}$
C
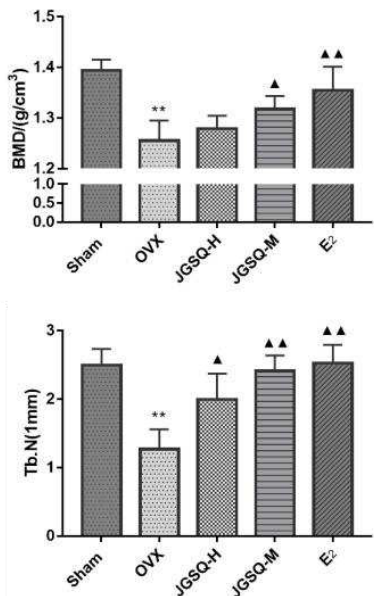
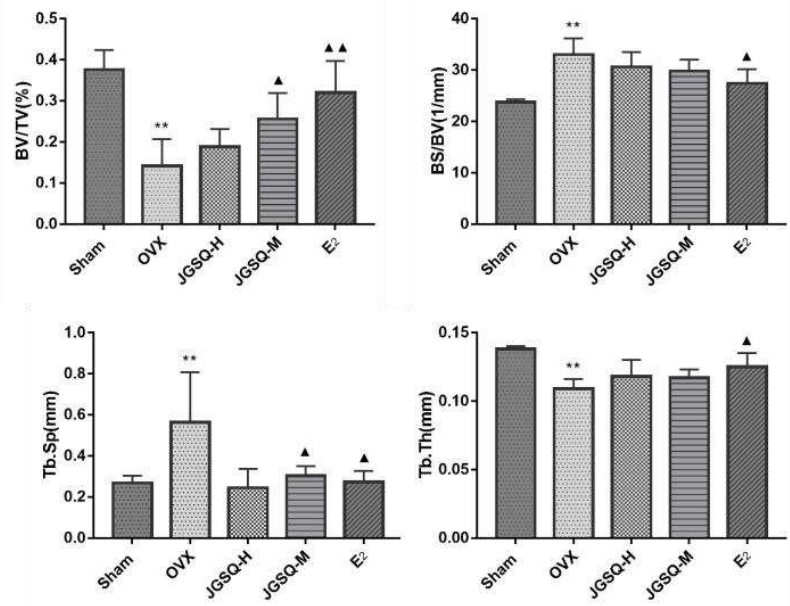

Figure.5 Effect of Jingui Shenqi Pills on Bone Mineral Density and Microstructure in Ovariectomized Rats. A. Representative 2D images of the vertical plane of the bone microstructure of the right distal femur $(n=3)$. B. Effects of JGSQ pills on the structural thickness of the right distal femur as displayed on 3D models. C. Effect of Jingui Shenqi pills on bone mineral density in ovariectomized rats. including BMD ( bone density) BV/TV (Bone Volume/Total Volume), BS/BV (Bone Surface/Bone Volume), Tb.N (trabecular number), Tb.Sp (trabecular separation) and $\mathrm{Tb} . \mathrm{Th}$ (trabecular thickness). *represents a comparison with the sham group, ${ }^{*} P<0.05,{ }^{* *} P<0.01$; ${ }^{\mathbf{\Delta}}$ represents a comparison with OVX group, ${ }^{\mathbf{\Delta}} P<0.05,{ }^{\mathbf{\Delta}} \boldsymbol{\Delta} P<0.01$.

\subsection{Expression of mRNA in bone tissue}

The expression of BMP-2, Smad1, and Runx2 mRNA in bone tissue was significantly decreased in the OVX group than the Sham group. However, the expression of BMP-2, Smad1 and Runx2 mRNA in bone tissue in the Jingui Shenqi pills treated group and E2 treated group was higher than in the OVX group (Fig.6A).

\subsection{Protein expression in bone tissue}


Western Blot analysis showed that the expression of BMP-2, Smad1, and Runx2 protein in bone tissue in the OVX group was significantly lower than in the Sham group, while the expression of BMP-2, Smad1, and Runx2 protein in bone tissue in Jingui Shenqi pills treated group and E2 treated group was higher than in the OVX group $(\mathrm{P}<0.05)$. There was no significant difference between the treatment groups (Fig.6B-6C).

A

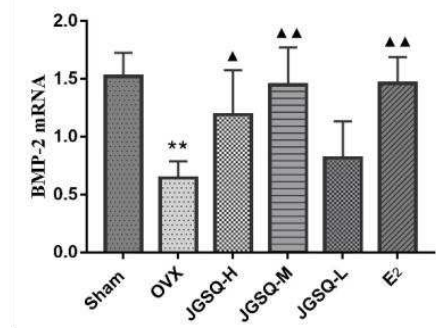

B

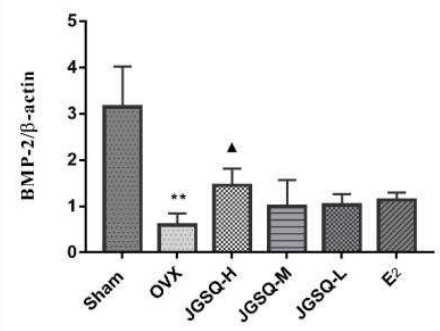

BMP-2

Smad1

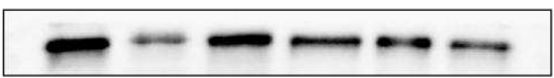

Runx2

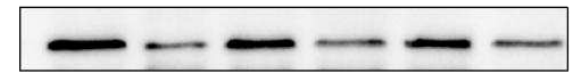

$\beta$-actin
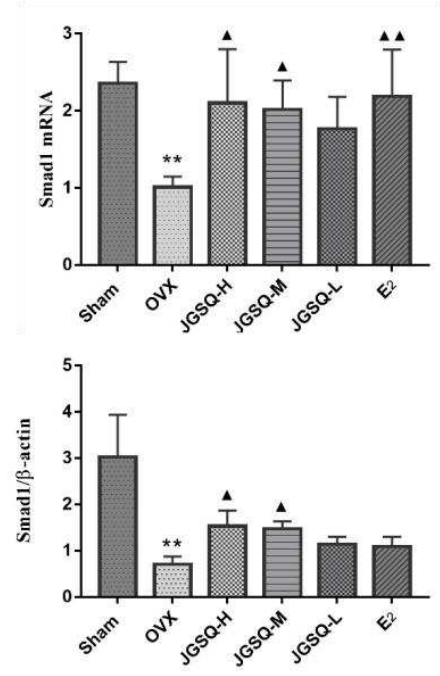
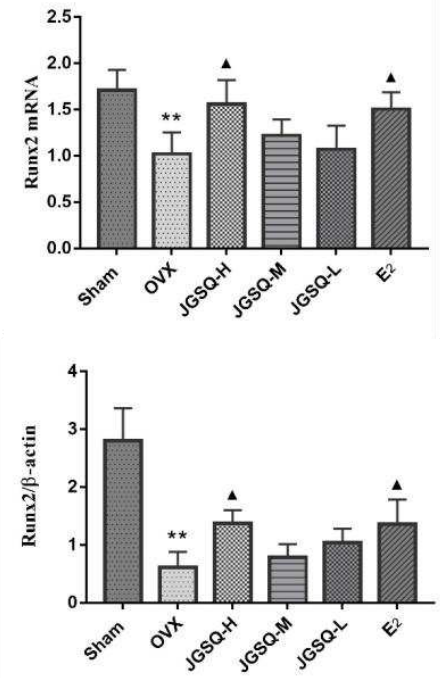

$45 \mathrm{kDa}$

$52 \mathrm{kDa}$

$67 \mathrm{kDa}$

42kDa

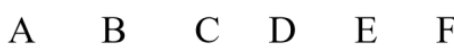

Figure.6 Effect of Jingui Shenqi Pills on BMP-2, Smad1, Runx2 gene expression in bone tissue of ovariectomized rats. A. The mRNA expression of BMP-2, Smad1 and Runx2 in rat bone tissues was measured by qRT-PCR. B. Western blot was used to detect the protein expression of BMP-2, Smad1 and Runx2 in rat bone tissues. C. Representative WB photographs of BMP-2, Smad1 and Runx2 were viewed, and $\beta$-actin was used for normalization.*represents a comparison with the sham group, ${ }^{*} P<0.05,{ }^{*} P<0.01 ;{ }^{\star}$ represents a comparison with OVX group, ${ }^{\mathbf{\Delta}} P<0.05, \mathbf{\Delta} \mathbf{\Delta} P<0.01$.

\section{Discussion}

The majority of the middle-aged and elderly people have a Yang deficiency. They often experience symptoms such as sore waist and knees, fear of cold, tinnitus, and diarrhea. Kidney 
yang is the foundation of the whole body yang. A deficiency in kidney yang results in bones lacking normal qi and blood nourishment, failure of joints to flex and extend normally, and impaired support resulting in osteoporosis. Jingui Shenqi pills prescription is derived from the synopsis of the Golden Chamber and is known as "the first prescription tonic for kidney yang through the ages". Jingui Shenqi pills can invigorate the kidney yang, gasify and return to normal, replenish the kidney yang, smooth the blood flow, and nourish the muscles, veins, and bones[22]. Qi Jicong found that Jingui Shenqi pills can relieve the pain caused by hyperosteogeny of the calcaneus and effectively treat "bone impotence"[23]. Jingui Shenqi pills were also reported to increase bone mineral density[24]. Fructus Corni Neoglycoside I has also been shown to promote the proliferation and differentiation of osteoblasts[25], and significantly increase the mRNA expression levels of Wnt2, BMP2, $\beta$-catenin, OPG, and NOX4 in osteoblasts[26]. Additionally, dried Rehmannia glutinosa has been shown to significantly inhibit the effects of OVX on the femur and lumbar vertebrae in rats[27].

The decline of ovarian function in menopause and the sudden drop of estrogen levels leads to a fat metabolism disorder, hyper appetite, and decreased activity, which reduces the basal metabolic rate and increases the fat content resulting in obesity[28] [29]. Excessive differentiation of adipocytes reduces osteoblast differentiation and bone formation, resulting in reduced bone formation and increased bone resorption, thus a decrease in bone mineral content, and osteoporosis[30] [31]. This study revealed that Jingui Shenqi pills reduce the weight gain induced by ovariectomy, increases the trabecular volume, and thickness, reduces trabecular separation, increases bone mineral density, and prevent the occurrence of postmenopausal osteoporosis.

Changes in the levels of calcium and phosphorus are important indicators that reflect on bone metabolism and changes to the whole body[32] [33] [34]. ALP (an intracellular enzyme in osteoblasts), is an important marker of bone formation[35]. When bone metabolism is exuberant, osteoblasts are active, and the ALP activity is increased. Estrogen secretion is decreased in OVX rats, resulting in a high bone metabolic state, that is, bone formation is less than bone resorption, simulating the bone metabolism in postmenopausal women[36]. OPG is secreted by osteoblasts and vascular endothelial cells and inhibits the differentiation and maturation of osteoclast precursors. Therefore, the level of serum OPG plays an important role in the early diagnosis of 
osteoporosis[37] [38]. Jingui Shenqi pills regulate bone metabolism and balance bone remodeling by increasing the levels of serum calcium, phosphorus, and OPG, and reducing the level of serum ALP.

Bone morphogenetic protein (BMP) is a family of proteins with multiple biological functions, which binds receptors on the target cell membrane. This results in activation of the BMP/Smad signaling transduction pathway[39]. The BMP-2 ligand secreted by osteocytes binds to the receptors BMPRI and BMPRII on the cell membrane forming a complex. The phosphorylated type II receptor activates the type I receptor, which then activates and phosphorylates receptor-regulated Smads protein $(\operatorname{Smad1} / 5 / 8)$ The regulated smads then bind to Smad4 and translocates to the nucleus. The complex binds to DNA binding protein and regulates the expression of downstream transcription factors Runx2 and OSX, thus regulating the transcription of genes related to different stages of osteogenic differentiation[16].

$\mathrm{BMP} / \mathrm{Smad}$ signaling pathway is a classical pathway in promoting osteoblast proliferation and differentiation in bone metabolism[40]. BMPs are an important osteogenic factor in inducing BMSCs to differentiate into osteoblasts and may result in increased osteoblast ALP activity, collagen synthesis, nerve cell differentiation, and kidney development[41] [42]. Smad protein mainly regulates bone metabolism through TGF- $\beta$ / BMP pathway, which directly or indirectly induces the proliferation, differentiation, and apoptosis of osteoblasts and osteoclasts[43] [44]. BMP/Smad signal pathway wholly regulates osteogenic differentiation, thus regulating osteogenic metabolism[45]. The study findings revealed a decrease in mRNA and protein levels of BMP-2, Smad1, and Runx2 in the OVX group compared to the sham group. Treatment with a high, medium, and low dose of Jingui Shenqi pills, increased mRNA and protein levels of BMP-2, Smad1, and Runx2. This proves that Jingui Shenqi pills are effective in preventing and treating PMOP by activation of the BMP/Smad signaling pathway. Jingui Shenqi pills also promote bone formation and improve osteoporosis via regulation of the BMP/Smad signaling pathway and increasing the expression of BMP-2, Smad1, and Runx2.

\section{Conclusions}


This study revealed that JGSQ promote bone formation, improves bone tissue microstructure, and increases bone mineral density by regulating the expression of the BMP/Smad signaling pathway. JGSQ offer clinical value in resolving osteoporosis. As a traditional Chinese medicine compound preparation with high safety and efficacy, JGSQ can be used as a preventive and therapeutic drug for osteoporosis for in-depth mining and development.

\section{Abbreviation:}

JGSQ-H, high-dose JGSQ pills; JGSQ-M, medium-dose JGSQ pills; JGSQ-L, Low-dose JKSQ pills; $E_{2}$, Estradilo; ELISA, enzyme linked immunosorbent assay; qRT-PCR, quantitative RTPCR; ALP, Alkaline Phosphatase; OPG, Osteoprotegerin; BMP-2, Bone Morphogenetic Protein 2.

\section{Ethics approval and consent to participate}

This experiment was reviewed by the Ethical Review Committee of Experimental Animal Welfare of Shandong University of traditional Chinese Medicine, and met the welfare and ethical requirements of experimental animals and animals (Batch number: SDUTCM2018112901).

\section{Consent for publication}

Not applicable.

\section{Availability of data and materials}

The data used to support the results of this study can be obtained from the frst author upon reasonable request.

\section{Competing interests}

The authors declare no conflict of interest.

\section{Funding}

This project was supported by the Youth Science Foundation of the National Natural Science Foundation of China (82004087), the Science and Technology Innovation team of Shandong 
University of Traditional Chinese Medicine (2020-54-19), and the Science and Technology Development Plan of Shandong Province of traditional Chinese Medicine (2017-004).

\section{Authors' contribution}

Huaxin Wang is fully responsible for experimental design, paper drafting and finalization. Qian Zhang and Xu Yang contributed equally to this manuscript.Qian Zhang, Xu Yang, Jin Wang, and $\mathrm{Xu}$ Tian completed the experiment and carried out statistical analysis. Ke Ma, Yanan Zhang, Yuan Wang and Xiaochun Han put forward constructive suggestions for the article, and Shijun Wang put forward important suggestions for the experimental process and the paper.All authors have read and approved the final version of the manuscript.

\section{Acknowledgements}

We are grateful the support from the Shandong Co-Innovation Center of Classic TCM Formula. 


\section{References}

1. Ma YZ, Wang YM, Liu Q, Li CL, Ma X, et al. 2018 China Guideline for the Diagnosis and Treatment of Senile Osteoporosis. Chinese Journal of Osteoporosis. 2019;39 (11):2557-2575.

2. Gillespie CW, Morin PE. Osteoporosis-Related Health Services Utilization Following First Hip Fracture Among a Cohort of Privately-Insured Women in the United States, 2008-2014: An Observational Study. 2017;32 (5):1052-1061.

3. Kanis JA, Burlet N, Cooper C, Delmas PD, Reginster JY, et al. European guidance for the diagnosis and management of osteoporosis in postmenopausal women. Osteoporosis International. 2008;19 (4):399-428.

4. Sheer RL, Barron RL, Sudharshan L, Pasquale MK, Ma X. Validated prediction of imminent risk of fracture for older adults. Am J Manag Care. 2020;26 (3):e91-e97.

5. Khan A, Fortier M, group Maow. Osteoporosis in menopause. Obstet Gynaecol Can. 2014;36 (9):839-840.

6. Manolagas SC, O'Brien CA, Almeida M. The role of estrogen and androgen receptors in bone health and disease. Nat Rev Endocrinol. 2013;9 (12):699-712.

7. Zhao F, Guo L, Wang X, Zhang Y. Correlation of oxidative stress-related biomarkers with postmenopausal osteoporosis: a systematic review and meta-analysis. Archives of Osteoporosis. 2021;16 (1):4-13.

8. Liu G-F, Wang Z-Q, Liu L, Zhang B-T, Miao Y-Y, et al. A network meta-analysis on the short-term efficacy and adverse events of different anti-osteoporosis drugs for the treatment of postmenopausal osteoporosis. 2018;119 (6):4469-4481.

9. Zhang Q, Wang HX, Wang Y, Liu LQ. Research progress in prevention and treatment of postmenopausal osteoporosis with traditional Chinese medicine. Chinese Journal of Osteoporosis. 2020;26 (07):1083-1088.

10. Huang LL, Zhu YJ, Lai XQ, Ju BL, Wang JH. Effects of Jinkui Shenqi Pill on Protein Expression in Femoral Cancellous Bone Tissues of Rats with Kidney-yang Deficiency Syndrome. Journal of traditional Chinese Medicine. 2018;59 (21):1862-1867.

11. Long YL, Li ZM. Effect of jingui shenqi pill and its disassembled recipes on ovarian functions in shen yang deficiency female rats. Zhongguo Zhong Xi Yi Jie He Za Zhi. 2021;33 (7):967971.

12. $\mathrm{Xu} \mathrm{CP}, \mathrm{Zhu} \mathrm{QJ}$, Song J, Li Z, Zhang D. Acceleration of Jingui Shenqi Pill on the testis telomerase activity in mice of Shen-yang deficiency. Zhongguo Zhong Xi Yi Jie He Za Zhi. 2013;33 (2):252-255.

13. Zhang BB, Dong KF. A meta-analysis of efficacy of two kinds of the Bushen medicine on BMD and VAS in osteoporosis patients. Clinical research of traditional Chinese medicine. 2020;12 (02):139-143.

14. Qiu WH. Comparison of the effects of Jinkui Shenqi Pill and Alendronate in the treatment of Postmenopausal Osteoporosis on Serum Osteoscleroprotein and Bone Mass. Journal of Clinical rational Drug use. 2019;12 (17):81-82.

15. Xu SJ, Huang JF, Shao M, Wang DT, Sun YQ. Effects and Potential Mechanisms of Bushen Recipes on Postmenopausal Osteoporosis. New drugs of traditional Chinese Medicine and Clinical Pharmacology. 2017;28 (05):588-593.

16. Wang HX. The Mechanism research of miR-5100 and formular of traditional Chinese Medicine in Osteogensis differentiation. Shandong University of traditional Chinese Medicine 2016.

17. Dong M, Jiao G, Liu H, Wu W, Li S, et al. Biological Silicon Stimulates Collagen Type 1 and Osteocalcin Synthesis in Human Osteoblast-Like Cells Through the BMP-2/Smad/RUNX2 Signaling Pathway. Biological Trace Element Research. 2016;173 (2):306-315.

18. Cheng Q, Tang W, Sheu T-J, Du Y, Gan J, et al. Circulating TGF- $\beta 1$ levels are negatively correlated with sclerostin levels in early postmenopausal women. Clinica Chimica Acta. 2016;455:87-92.

19. Lademann F, Weidner H, Tsourdi E, Kumar R, Rijntjes E, et al. Disruption of BMP Signaling Prevents Hyperthyroidism-Induced Bone Loss in Male Mice. Journal of Bone and Mineral Research. 2020;35 (10):2058-2069.

20. Cui Q, Xing JH, Yu M, Wang Y, Xu J. Mmu-miR-185 depletion promotes osteogenic differentiation and suppresses bone loss in osteoporosis through the Bgn-mediated BMP/Smad pathway. Cell Death Dis. 2019;10 (3):172. 
21. Li ZW. Effect of Kidney-tonifying and Bone-strengthening Therapy on the expression of BMP2, Smad1/5 and Smad6 in femur of Rats with Osteoporosis: Hubei Institute for nationalities; 2018.

22. Li S, Li D. Clinical application and analysis of Jinkui Shenqi Pill.Chinese Journal of traditional Chinese Medicine. 2015;30 (03):928-930.

23. Qi JC, Yu D. Observation of Efficacy of Modified Jinkui Shenqi Pill in Combination with Physical Therapy on Calcaneal Bone Hyperplasia.Henan traditional Chinese medicine. 2018;38 (09):1315-1318.

24. Zeng Q. Clinical Treatment of Osteoporosis in Traditional Chinese Medicine.Shenzhen Journal of Integrated traditional Chinese and Western Medicine. 2016;26 (19):54-55.

25. Park E, Lee CG, Kim J, Lim E, Hwang S, et al. Anti-Osteoporotic Effects of the Herbal Mixture of Cornus officinalis and Achyranthes japonica In Vitro and In Vivo. Plants (Basel). 2020;9 (9):1114.

26. Huang JC, Lin YP, Chen TY, Chai S, Huang HX. Effect of cornuside I on the proliferation and osteogenic differentiation of osteoblasts.Chinese Journal of Osteoporosis. 2020;26 (01):15.

27. Lim DW, Kim YT. Dried root of Rehmannia glutinosa prevents bone loss in ovariectomized rats. Molecules. 2013;18 (5):5804-5813.

28. Ko S-H, Kim H-S. Menopause-Associated Lipid Metabolic Disorders and Foods Beneficial for Postmenopausal Women. Nutrients. 2020;12 (1):202.

29. Yoon M. The role of PPAR $\alpha$ in lipid metabolism and obesity: Focusing on the effects of estrogen on PPAR $\alpha$ actions. Pharmacological Research. 2009;60 (3):151-159.

30. You L, Pan L, Chen L, Gu W, Chen J. MiR-27a is Essential for the Shift from Osteogenic Differentiation to Adipogenic Differentiation of Mesenchymal Stem Cells in Postmenopausal Osteoporosis.Cell Physiol Biochem. 2026;39 (1):253-265.

31. Yu B, Huo LH, Liu YS, Deng P, John S. PGC-1 $\alpha$ Controls Skeletal Stem Cell Fate and BoneFat Balance in Osteoporosis and Skeletal Aging by Inducing TAZ.Cell stem cell. 2018;23 (2):193-209.e195.

32. Watcho P, Guiadem Kamto Kamto B, Defo Deeh PB, Nguelefack TB, Kamanyi A, et al. The aqueous and methanol extracts of Bambusa vulgaris (Poaceae) improve calcium and phosphorus levels, and bone microstructure in ovariectomized model of osteoporosis. Journal of Basic and Clinical Physiology and Pharmacology. 2019;30 (3).

33. Bansal N, Katz R, Boer IHd, Kestenbaum B, Siscovick DS. Influence of estrogen therapy on calcium, phosphorus, and other regulatory hormones in postmenopausal women: the MESA study.J Clin Endocrinol Metab. 2013;98 (12):4890-4898.

34. Redmond J, Jarjou LMA, Zhou B, Prentice A, Schoenmakers I. Ethnic differences in calcium, phosphate and bone metabolism.Proc Nutr Soc. 2014;73 (2):340-351.

35. Atalay S, Elci A, Kayadibi H, Onder CB, Aka N. Diagnostic utility of osteocalcin, undercarboxylated osteocalcin, and alkaline phosphatase for osteoporosis in premenopausal and postmenopausal women.Ann Lab Med. 2012;32 (1):23-30.

36. Mukaiyama K, Kamimura M, Uchiyama S, Ikegami S, Nakamura Y, et al. Elevation of serum alkaline phosphatase (ALP) level in postmenopausal women is caused by high bone turnover. Aging clinical and experimental research. 2015;27 (4):413-418.

37. Tang H, Zhu XW, Wu LF, Mo XB, Deng FY. Integrative Analysis Confirmed the Association between Osteoprotegerin and Osteoporosis.Chin Med Sci J. 2019;34 (2):147-156.

38. Hamdy NA. Osteoprotegerin as a potential therapy for osteoporosis. Curr Osteoporos Rep. 2005;3 (4):121-125.

39. Wang GL, Cai J, Zhang JS, Li CY. Mechanism of triptolide in treating ankylosing spondylitis through the antiossification effect of the BMP/Smad signaling pathway. Molecular medicine reports. 2018;17 (2).

40. An XN. Study on the mechanism of PNS-mediated BMP-Smad signal pathway regulating osteogenic differentiation of BMSCs: Guangxi Medical University; 2019.

41. Ikushima H, Miyazono K. TGF- $\beta$ signal transduction spreading to a wider field: a broad variety of mechanisms for context-dependent effects of TGF- $\beta$. Cell and Tissue Research. 2012;347 (1):37-49.

42. Raje MM, Ashma R. Epigenetic regulation of BMP2 gene in osteoporosis: a DNA methylation study. Molecular Biology Reports. 2019;46 (2):1667-1674. 
43. Gregson CL, Bergen DJM, Leo P, Sessions RB, Wheeler L. A Rare Mutation in SMAD9 Associated With High Bone Mass Identifies the SMAD-Dependent BMP Signaling Pathway as a Potential Anabolic Target for Osteoporosis.J Bone Miner Res. 2020;1 (35):92-105.

44. Liu J, Lu CW, Wu XH, Zhang ZK, Li J. Targeting osteoblastic casein kinase-2 interacting protein-1 to enhance Smad-dependent BMP signaling and reverse bone formation reduction in glucocorticoid-induced osteoporosis. Scientific reports. 2017;7:41295-41295.

45. Chai S, Wan L, Wang J-L, Huang J-C, Huang H-X. Gushukang inhibits osteocyte apoptosis and enhances BMP-2/Smads signaling pathway in ovariectomized rats \% $\mathrm{J}$ Phytomedicine. $2019 ; 64$. 


\section{Figure Legends}

Figure.1 Total ion flow patterns of JGSQ pills in positive (A) and negative ion (B) modes.

Figure.2 The general condition of rats. A. Effect of Jingui Shenqi pills on rats body weight. B. Body weight of rats in each group at Week 12. C. Effect of Jingui Shenqi pills on uterine coefficient different rat groups. *represents a comparison with the sham group, ${ }^{*} P<0.05, * * P<$ $0.01 ;{ }^{\star}$ represents a comparison with OVX group, ${ }^{\wedge} P<0.05,{ }^{\wedge} \Delta P<0.01$.

Figure.3 Effect of Jingui Shenqi Pills on Serum Parameters in Ovariectomized Rats. A,B. Effect of Jingui Shenqi pills on calcium and phosphorus in serum of ovariectomized rats. C,D. Effect of Jingui Shenqi pills on serum ALP and OPG in ovariectomized rats. *represents a comparison with the sham group, $* P<0.05, * * P<0.01$; ${ }^{\star}$ represents a comparison with OVX group, $\mathbf{\Delta} P<0.05, \boldsymbol{\Delta} \Delta P<0.01$.

Figure.4 Effect of Jingui Shenqi Pills on Pathological Changes of Femur in Ovariectomized Rats.Representative photographs of H\&E stained femoral sections from each group $(\times 100)$. Arrow (black): bone.

Figure.5 Effect of Jingui Shenqi Pills on Bone Mineral Density and Microstructure in Ovariectomized Rats. A. Representative 2D images of the vertical plane of the bone microstructure of the right distal femur $(n=3)$. B. Effects of JGSQ pills on the structural thickness of the right distal femur as displayed on 3D models. C. Effect of Jingui Shenqi pills on bone mineral density in ovariectomized rats. including BMD ( bone density) BV/TV (Bone Volume/Total Volume), BS/BV (Bone Surface/Bone Volume), Tb.N (trabecular number), Tb.Sp (trabecular separation) and Tb.Th (trabecular thickness). *represents a comparison with the sham group, $* P<0.05, * * P<0.01 ;{ }^{\wedge}$ represents a comparison with OVX group, $\Delta P<0.05, \wedge \mathbf{\Delta} P<0.01$.

Figure.6 Effect of Jingui Shenqi Pills on BMP-2, Smad1, Runx2 gene expression in bone tissue of ovariectomized rats. A. The mRNA expression of BMP-2, Smad1 and Runx2 in rat bone tissues was measured by qRT-PCR. B. Western blot was used to detect the protein expression of BMP-2, Smad1 and Runx2 in rat bone tissues. C. Representative WB photographs of BMP-2, Smad1 and Runx2 were viewed, and $\beta$-actin was used for normalization.*represents a comparison with the sham group, $* P<0.05, * * P<0.01$; ${ }^{\star}$ represents a comparison with OVX group, $\Delta P<0.05, \Delta \wedge P<0.01$.

Figure.7 Graphical abstract: Jingui Shenqi pills Prevents and Treats Postmenopausal osteoporosis via Regulating the BMP/Smad Signaling Pathway. 
Table 1 HPLC gradient elution process

\begin{tabular}{ccc}
\hline Time (min) & Mobile phase A & Mobile phase B \\
\hline 0 & $98 \%$ & $2 \%$ \\
\hline $0-5$ & $98-80 \%$ & $2-20 \%$ \\
\hline $5-10$ & $80-50 \%$ & $20-50 \%$ \\
\hline $10-15$ & $50-20 \%$ & $50-80 \%$ \\
\hline $15-20$ & $20-5 \%$ & $80-95 \%$ \\
\hline $20-25$ & $5 \%$ & $95 \%$ \\
\hline $25-30$ & $5-98 \%$ & $95-2 \%$ \\
\hline
\end{tabular}

Table 2: The primer sequences

\begin{tabular}{|c|c|c|}
\hline Primer & & Sequence of primers \\
\hline \multirow[t]{2}{*}{ ВМР-2 } & Forward & 5'-TGCGGTCTCCTAAAGGTCG-3' \\
\hline & Reverse & 5'-ACTCAAACTCGCTGAGGACG-3' \\
\hline \multirow[t]{2}{*}{ Smad1 } & Forward & 5'-TCAATAGAGGAGATGTTCAAGCAGT-3' \\
\hline & Reverse & 5'-AAACCATCCACCAACACGCT-3' \\
\hline \multirow[t]{2}{*}{ Runx2 } & Forward & 5'-GCCGGGAATGATGAGAACTA-3' \\
\hline & Reverse & 5'-GGTGAAACTCTTGCCTCGTC-3' \\
\hline \multirow[t]{2}{*}{$\beta$-actin } & Forward & 5'-TGTCACCAACTGGGACGATA-3' \\
\hline & Reverse & 5'-GGGGTGTTGAAGGTCTCAAA-3' \\
\hline
\end{tabular}


Table 3. Main components in JGSQ pills

\begin{tabular}{|c|c|c|c|c|c|}
\hline No. & $\begin{array}{c}\text { Assigned } \\
\text { identity }\end{array}$ & $\begin{array}{c}\text { Molecular } \\
\text { formula }\end{array}$ & $\begin{array}{c}\text { Molecular } \\
\text { weight }\end{array}$ & $\begin{array}{c}\text { TR } \\
(\min )\end{array}$ & Area (Max.) \\
\hline 1 & Salsolinol & $\mathrm{C}_{10} \mathrm{H}_{13} \mathrm{NO}_{2}$ & 179.09433 & 1.876 & 146442.6449 \\
\hline 2 & L-Serin & $\mathrm{C}_{3} \mathrm{H}_{7} \mathrm{NO}_{3}$ & 105.04288 & 1.252 & 140976.2545 \\
\hline 3 & choline & $\mathrm{C}_{5} \mathrm{H}_{13} \mathrm{NO}$ & 103.09997 & 1.114 & 573034.5195 \\
\hline 4 & uracil & $\mathrm{C}_{4} \mathrm{H}_{4} \mathrm{~N}_{2} \mathrm{O}_{2}$ & 112.02746 & 2.876 & 54295.12559 \\
\hline 5 & gallic acid & $\mathrm{C}_{7} \mathrm{H}_{6} \mathrm{O}_{5}$ & 170.02036 & 4.759 & 679165.5473 \\
\hline 6 & mussaenosidic acid & $\mathrm{C}_{16} \mathrm{H}_{24} \mathrm{O}_{10}$ & 376.13643 & 6.271 & 49487.24167 \\
\hline 7 & caffeic acid & $\mathrm{C}_{9} \mathrm{H}_{8} \mathrm{O}_{4}$ & 180.04187 & 7.01 & 261110.5383 \\
\hline 8 & Loganin & $\mathrm{C}_{17} \mathrm{H}_{26} \mathrm{O}_{10}$ & 390.15153 & 7.646 & 319772.86 \\
\hline 9 & $\begin{array}{l}\text { 1,2,3,6-tetra-O-galloyl- } \beta \text {-D- } \\
\text { glucose }\end{array}$ & $\mathrm{C}_{34} \mathrm{H}_{28} \mathrm{O}_{22}$ & 788.10678 & 8.579 & 342536.3267 \\
\hline 10 & Myristicin & $\mathrm{C}_{11} \mathrm{H}_{12} \mathrm{O}_{3}$ & 192.07852 & 9.33 & 129656.2855 \\
\hline 11 & trans-cinnamic acid & $\mathrm{C}_{9} \mathrm{H}_{8} \mathrm{O}_{2}$ & 148.0512 & 10.573 & 63330.4939 \\
\hline 12 & coumarin & $\mathrm{C}_{9} \mathrm{H}_{6} \mathrm{O}_{2}$ & 146.03653 & 10.624 & 499750.233 \\
\hline 13 & abscisic acid & $\mathrm{C}_{15} \mathrm{H}_{20} \mathrm{O}_{4}$ & 264.13596 & 10.779 & 65729.18049 \\
\hline 14 & quercetin & $\mathrm{C}_{15} \mathrm{H}_{10} \mathrm{O}_{7}$ & 302.04227 & 11.018 & 369557.0775 \\
\hline 15 & (-)-Caryophyllene oxide & $\mathrm{C}_{15} \mathrm{H}_{24} \mathrm{O}$ & 220.18231 & 11.518 & 141614.446 \\
\hline 16 & methyl palmitate & $\mathrm{C}_{17} \mathrm{H}_{34} \mathrm{O}_{2}$ & 287.28184 & 11.572 & 907368.2794 \\
\hline \multirow[t]{2}{*}{17} & 3-[2- $(3-$ & & & & \\
\hline & $\begin{array}{l}\text { hydroxyphenyl)ethyl]-5- } \\
\text { methoxyphenol }\end{array}$ & $\mathrm{C}_{15} \mathrm{H}_{16} \mathrm{O}_{3}$ & 244.10948 & 12.956 & 398562.2315 \\
\hline 18 & diosgenin & $\mathrm{C}_{27} \mathrm{H}_{42} \mathrm{O}_{3}$ & 396.30184 & 16.115 & 86761.9485 \\
\hline 19 & oleanolic acid & $\mathrm{C}_{30} \mathrm{H}_{48} \mathrm{O}_{3}$ & 438.34867 & 19.55 & 457465.9619 \\
\hline 20 & ursolic acid & $\mathrm{C}_{30} \mathrm{H}_{48} \mathrm{O}_{3}$ & 456.35977 & 19.572 & 251869.2106 \\
\hline 21 & oleic acid & $\mathrm{C}_{18} \mathrm{H}_{34} \mathrm{O}_{2}$ & 282.25569 & 21.301 & 449661.2603 \\
\hline 22 & Stearamide & $\mathrm{C}_{18} \mathrm{H}_{37} \mathrm{NO}$ & 283.28693 & 21.43 & 176398.883 \\
\hline 23 & stearic acid & $\mathrm{C}_{18} \mathrm{H}_{36} \mathrm{O}_{2}$ & 284.27136 & 22.845 & 148504.1647 \\
\hline 24 & Erucamide & $\mathrm{C}_{22} \mathrm{H}_{43} \mathrm{NO}$ & 337.33348 & 23.63 & 1440974.297 \\
\hline
\end{tabular}

